\title{
Nuevos registros de Cycadales y Cycadeoidales del Triásico superior del río Biobío, Chile
}

\author{
New records of Upper Triassic Cycadales and Cycadeoidales of Biobío river, Chile
}

\author{
MARCELO LEPPE \& PHILIPPE MOISAN
}

Departamento de Botánica, Facultad de Ciencias Naturales y Oceanográficas,

Universidad de Concepción, Casilla 160-C, Concepción, Chile; e-mail: mleppe@udec.cl

\begin{abstract}
RESUMEN
Se entrega un aporte al conocimiento de las Cycadales y Cycadeoidales presentes en los sedimentos del Triásico superior de la Formación Santa Juana (Cárnico-Rético) de la Región del Biobío, Chile. Los grupos están representados por las especies Pseudoctenis longipinnata Anderson \& Anderson, Pseudoctenis spatulata Du Toit y Pterophyllum azcaratei Herbst \& Troncoso, y se propone una nueva especie Pseudoctenis truncata nov. sp. Las especies se encuentran junto a otros elementos típicos de las asociaciones paleoflorísticas del borde suroccidental del Gondwana.
\end{abstract}

Palabras clave: Triásico superior, paleobotánica, Cycadales, Cycadeoidales.

\begin{abstract}
A contribution to the knowledge of Cycadales and Cycadeoidales present in the Upper Triassic of the Santa Juana Formation (Carnian-Raetian) in the Bio-Bío Region of Chile is provided. The groups are represented by the species Pseudoctenis longipinnata Anderson \& Anderson, Pseudoctenis spatulata Du Toit and Pterophyllum azcaratei Herbst \& Troncoso. A new species Pseudoctenis truncata nov. sp. is described. They appear to be related to other typical elements of the paleofloristic assamblages from the south-occidental border of Gondwanaland.
\end{abstract}

Key words: Upper Triassic, paleobotany, Cycadales, Cycadeoidales.

\section{INTRODUCCIÓN}

Frecuentemente se ha tratado a las Cycadeoidales (Bennettitales) y a las Cycadales como parte de las Cycadophyta. También han sido asignadas dos clases distintas dentro de las Pinophyta, o a grupos distintos como las Cycadophyta y Cycadeoidophyta. Ambos grupos comparten estructuras vegetativas muy similares, pero sus estructuras reproductivas (conos en el caso de las Cycadeoidales y estróbilos en el caso de las Cycadales) contrastan marcadamente entre sí. Esto sugiere que incluso podrían ser considerados como grupos evolutivamente distantes (Nishida 1994).

Actualmente el origen de ambos grupos también se considera un tanto diferente. Un posible antecesor de las Cycadeoidales puede ser hallado en las Medullosales paleozoicas, fundamentando su parentesco en similitudes que muestran a nivel de la estructura de la madera y de los patrones de ramificación de los haces vasculares (Stewart \& Rothwell 1993, Taylor \& Taylor 1993). Sin embargo, se reconoce la existencia de una brecha morfológica entre ambos grupos, ya que a diferencia de las Medullosales, las Cycadeoidales poseen un tallo anatómicamente bien diferenciado y hojas básicamente en frondas pinnadas (Crane 1985, Nishida 1994). Estudios de sus conos sugieren además a las Cycadeoidales como un posible ancestro de las angiospermas (Crane 1985). Por otro lado, el grupo remanente de las Cycadales actuales aparece como sobreviviente de un grupo mucho mayor que dominó parte de las floras mesozoi-

\footnotetext{
1 GNAEDINGER S (1999) La flora triásica del grupo El Tranquilo, Provincia de Santa Cruz, Patagonia. Parte VII: Cycadophyta. Actas del X Simposio Argentino de Paleobotánica y Palinología, Mendoza, Argentina: 27-32.

${ }^{2}$ LEPPE M, E ABAD \& L LEPEZ (2000) Aportes al conocimiento paleoflorístico de la Formación Santa Juana, Triásico superior de la VIII Región de Chile. Actas del IX Congreso Geológico Chileno, Puerto Varas, Chile 1: 257-259.
} 
cas, principalmente durante el Jurásico (Gifford \& Foster 1987) y que hallaría su origen en el Pérmico Inferior (Artabe \& Stevenson 1999).

El presente trabajo se basa en el estudio de representantes de ambos grupos presentes en los afloramientos plantíferos denominados informalmente como Formación Santa Juana $(\mathrm{Cu}-$ curella 1978), ubicados en la cuenca del bajo Biobío (localidades de Santa Juana, Quilacoya, Gomero, Calquinhue y Patagual) (Fig. 1). La edad Triásica superior (Fig. 2), fue asignada tentativamente por Steinmann (1921), la Formación Santa Juana se basa en la presencia de plantas fósiles de edad rética, tales como: $\mathrm{Cla}$ thropteris platyphylla Goeppert, Pterophyllum sp., Pecopteris cottoni Zeiller, Dicroidium odontopteroides Feistm, D. zuberi (Szajnocha) Archangelsky, Baiera sp. Nathorst y Linguifolium lilleanum Arber (Tavera 1960).

Solo breves trabajos han hecho alusión al contenido fosilífero del Triásico del río Biobío.
Estos afloramientos fueron descritos, en los primeros trabajos, como de origen continental a marino, con contenido de plantas y animales fósiles (Steinmann 1921). Jaworski (1922) asignó una edad cárnica a las rocas del área de Talcamávida en base a la presencia de invertebrados marinos, como Arcestes sp. Brüggen (1934), tras una revisión del material recolectado por Steinmann (1921) y Jaworski (1922), resumió sus resultados agregando nuevos reportes de afloramientos ubicados en la ruta ferroviaria entre San Rosendo y Gomero. Otros trabajos hacen mención a los estratos fosilíferos triásicos de Concepción (Groeber 1934, Gerth 1935, Kummel \& Fuchs 1953). Tavera (1960) tras una revisión más extensa que la de sus predecesores, dividió la secuencia en tres miembros, llamados: Miembro Quilacoya (continental), Miembro Unihue (marino) y Miembro Talcamávida-Gomero. Veyl (1961) en la geología regional de Concepción, basada en Brüggen

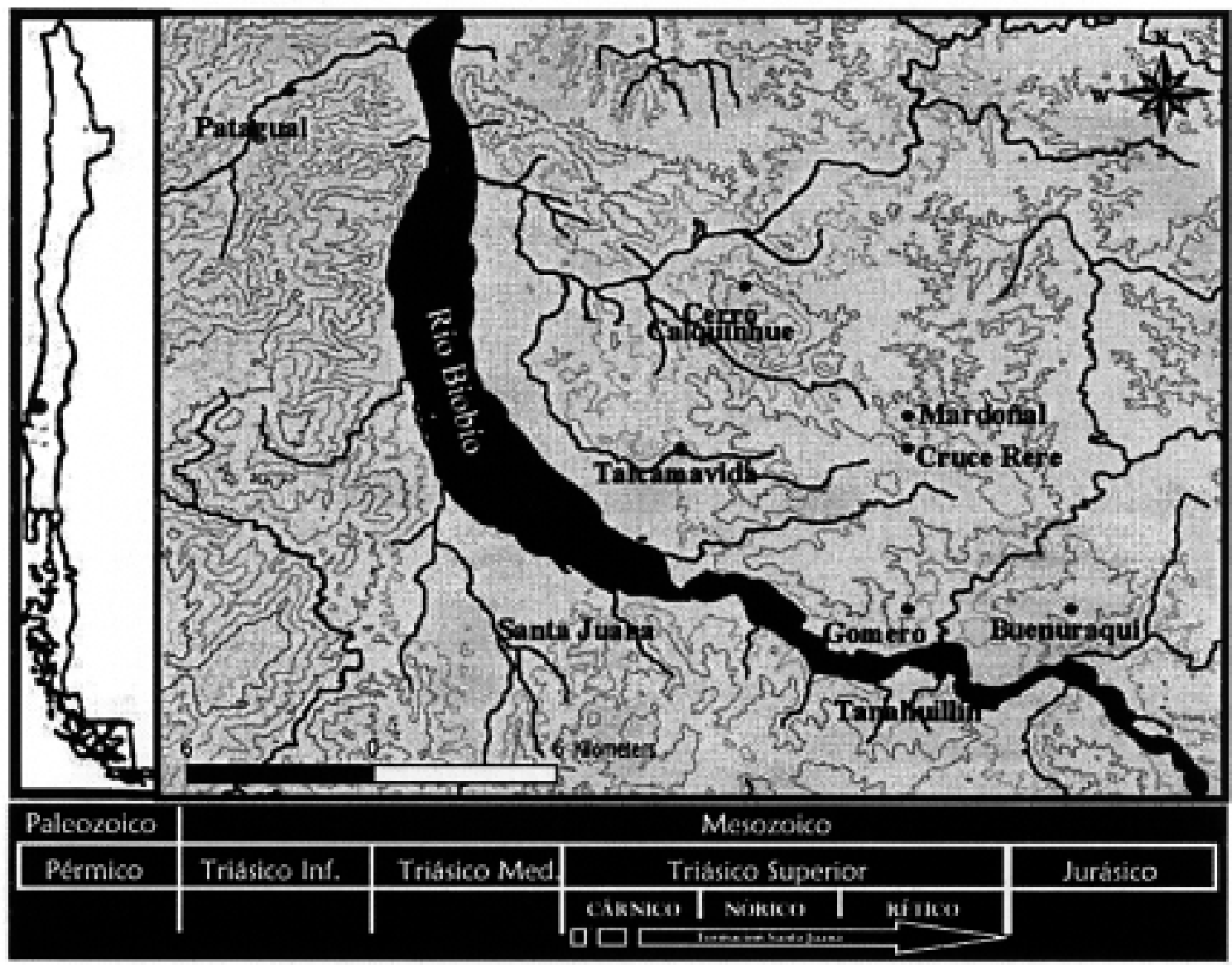

Fig. 1: Puntos de muestreo en la Región del Biobío. Abajo: escala de tiempo geológico, la flecha indica la edad relativa de los afloramientos estudiados.

Sampling points in the Biobío region. Below: geologic timescale, the arrow shows the relative age of the outcrops studied. 


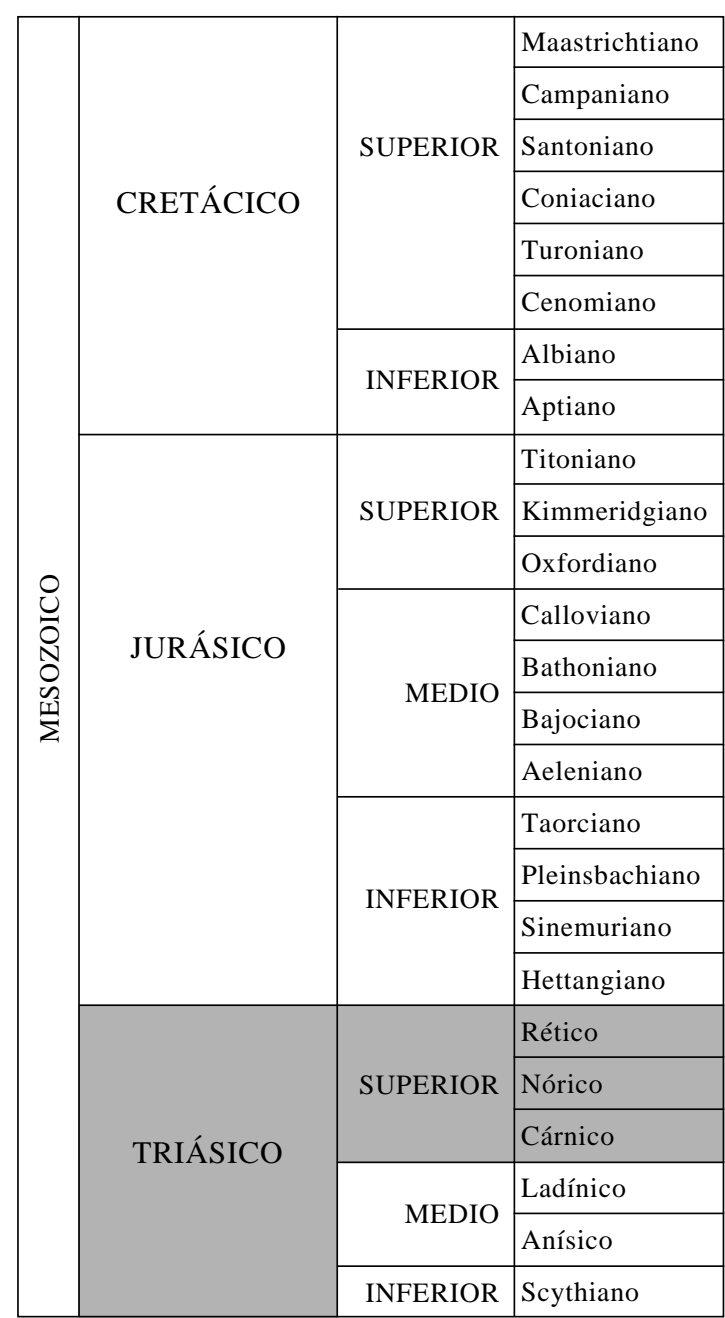

Fig. 2: Escala geológica de tiempo entre los 245 millones de años (comienzos del Scythiano) y los 65 millones de años (fines del Maastrichtiano). La zona destacada en gris representa la edad asignada a los estratos de la Formación Santa Juana.

Geological timescale between 245 million years ago (at the beginning of the Scythian) and 65 million years ago (end of Maastrichtian). The gray zone represents the age assigned to the Santa Juana Formation layers.

(1934), Steinmann (1921), Jaworsky (1922) y Tavera (1960). En 1983, Anderson \& Anderson (1983) señalan la presencia de representantes de Cycadales y Cycadeoidales en los afloramientos de Santa Juana. Dentro de este contexto, Tavera (1960) hace mención a Pterophyllum sp. basándose en muestras colectadas por Fuenzalida (1937) en Buenuraqui-Gomero; esta especie fue posteriormente asignada por Herbst \& Troncoso (2000) como Pterophyllum azcaratei. Otras menciones de los taxa referidos en este estudio la realiza Nielsen (1999), quien determinó Pseudoctenis fissa Du Toit, Pseudoctenis gracipinnata Anderson \& Anderson y Pseudoctenis santajuanaensis nov. sp.

En el presente estudio se evaluó la flora triásica de la VIII Región de Chile, con énfasis en las Cycadales y Cycadeoidales, y se establece su relación con otras floras sudamericanas y no sudamericanas.

\section{MATERIALES Y MÉTODOS}

El material estudiado corresponde a improntas de hojas procedentes de las localidades de Cerro Calquinhue, Gomero, Santa Juana y Patagual (Fig. 1). El sitio Gomero se ubica a $200 \mathrm{~m}$ al NW de la estación de ferrocarriles y en el sector de la Escuela de Gomero, Provincia de Concepción, VIII Región $\left(37^{\circ} 12,138\right.$ ' S; $72^{\circ} 47,665^{\prime}$ O). Edad Triásico superior. El sitio Cerro Calquinhue se ubica en el flanco oeste del Cerro, en el camino forestal "Cota 100" del sector Quebrada La Mina donde se ubican dos pirquenes carboníferos abandonados, Provincia de Concepción, VIII Región (37 $5,95^{\prime}$ S; 72 $53.534^{\circ}$ O). Edad Triásico superior.

El material colectado fue trasladado al $\mathrm{Mu}-$ seo del Departamento de Ciencias de la Tierra (CONC) de la Universidad de Concepción, donde fue descrito mediante el uso de una lupa Carl Zeiss y una cámara clara para la realización de diagramas. Las fotografías (72) fueron obtenidas mediante una cámara digital Sony Mavica con un zoom de 30x. Las improntas (25) quedaron almacenadas y etiquetadas como TriX 1-25, en el estante identificado como "Triásico y Paleozoico" de CONC. El holotipo de la especie nueva Pseudoctenis truncata se encuentra almacenado con el código TriX-2, en la colección del Laboratorio de Paleobotánica del Departamento Ciencias de la Tierra, Universidad de Concepción, Chile.

\section{RESULTADOS}

De las 21 muestras analizadas, doce corresponden a Pseudoctenis longipinnata (Anderson \& Anderson 1989), dos a Pseudoctenis spatulata (Herbst \& Troncoso 2000), seis a Pterophyllum azcaratei (Herbst y Troncoso 2000) y dos a Pseudoctenis truncata nov. sp.

\section{Taxonomía}

División Pinophyta Meyen, 1984.

Clase Cycadopsida Meyen, 1984.

Orden Cycadales Meyen, 1984. 


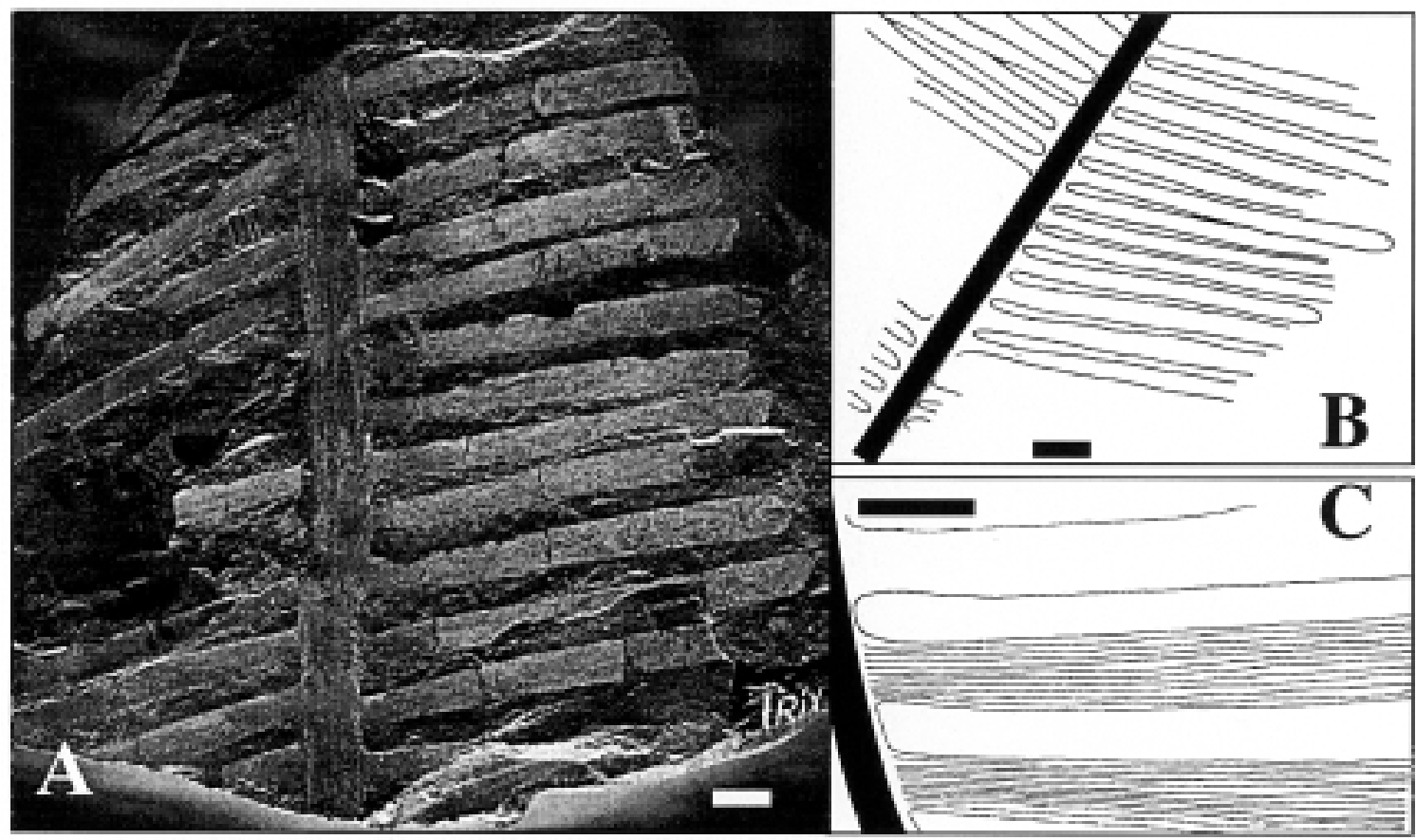

Fig. 3: (A, B) Pseudoctenis longipinnata Anderson \& Anderson (TriX 8), porción medial de la hoja, con pinnas de disposición opuesta y alternada (C), esquema de la venación simple, sin dicotomía. Barras de escala representan $1 \mathrm{~cm}$.

(A-B) Pseudoctenis longipinnata Anderson \& Anderson (TriX 8), medial portion of leaf, pinnae with alternate and opposite disposition; (C), diagram of the simple venation without dichotomy. Scale bars represent $1 \mathrm{~cm}$ length.

Género Pseudoctenis Seward, 1911:

Especie tipo P. eathiensis Seward, 1911.

Pseudoctenis longipinnata Anderson \& Anderson, 1989 (Fig. 3A-C). Material estudiado: TriX 1-12, hallado en las localidades de Gomero y Cerro Calquinhue. Descripción: frondas incompletas, donde los fragmentos más grandes llegan a medir $160 \mathrm{~mm}$ de largo por $110 \mathrm{~mm}$ de ancho, pero en general tienen una forma lanceolada, con la lámina aplastada dorsalmente. Las pinnas miden en promedio $50 \pm$ $10 \mathrm{~mm}$ de largo y entre 3 a $5 \mathrm{~mm}$ de ancho. El raquis frecuentemente mide de 4 a $6 \mathrm{~mm}$ de ancho. Las pinnas se disponen en ángulos de 75 a $85^{\circ}$ con respecto al eje del raquis, levemente acroscópicas en la porción proximal, mientras que las pinnas distales son fuertemente acroscópicas. Las pinnas se insertan levemente ensanchadas lo que produce la presencia de ala laminar, para continuar casi rectas y paralelas hasta un ápice redondeado y algo aguzado. Pinnas alternadas y opuestas, con una distancia entre ellas de 2 a $4 \mathrm{~mm}$. Las venas salen de toda la base, son paralelas, no se observa dicotomía. Aproximadamente de 11-13 venas por pinna.

Discusión: el material observado se ajusta plenamente con los caracteres diagnósticos enunciados por Anderson \& Anderson (1989) en lo que se refiere a la morfología macroscópica. La falta de conservación cuticular hace imposible la comparación microscópica. Igualmente las muestras aquí descritas comparten la morfología con las reportadas por Herbst \& Troncoso (2000) para el Triásico Superior de Quebrada La Cachivarita (Formación La Ternera). En la Región del Biobío aparece asociada a rocas del Nórico-Rético.

Clase Cycadopsida Meyen, 1984.

Orden Cycadales Meyen, 1984.

Pseudoctenis cf. spatulata Du Toit, 1927 (Fig. 4A-D): Sinonimia Pseudoctenis capensis Du Toit,1927. Material estudiado: TriX 19-20, recolectado en la Quebrada La Mina en el Cerro Calquinhue. Descripción: Fragmentos de frondas con las bases de las pinnas miden entre 3 a $4 \mathrm{~mm}$ de ancho, ensanchándose posteriormente hasta unos 8 o $9 \mathrm{~mm}$. Este carácter y la constricción basal provee a las pinnas una forma oblongo-alargada. Se observa levemente ala laminar. El raquis mide $4 \mathrm{~mm}$ de ancho. Las pinnas se insertan al raquis en ángulos de 65 a $75^{\circ}$. Pinnas de disposición opuesta y alternada, con una distancia entre ellas de 2,5 a $3 \mathrm{~mm}$ en la zona 

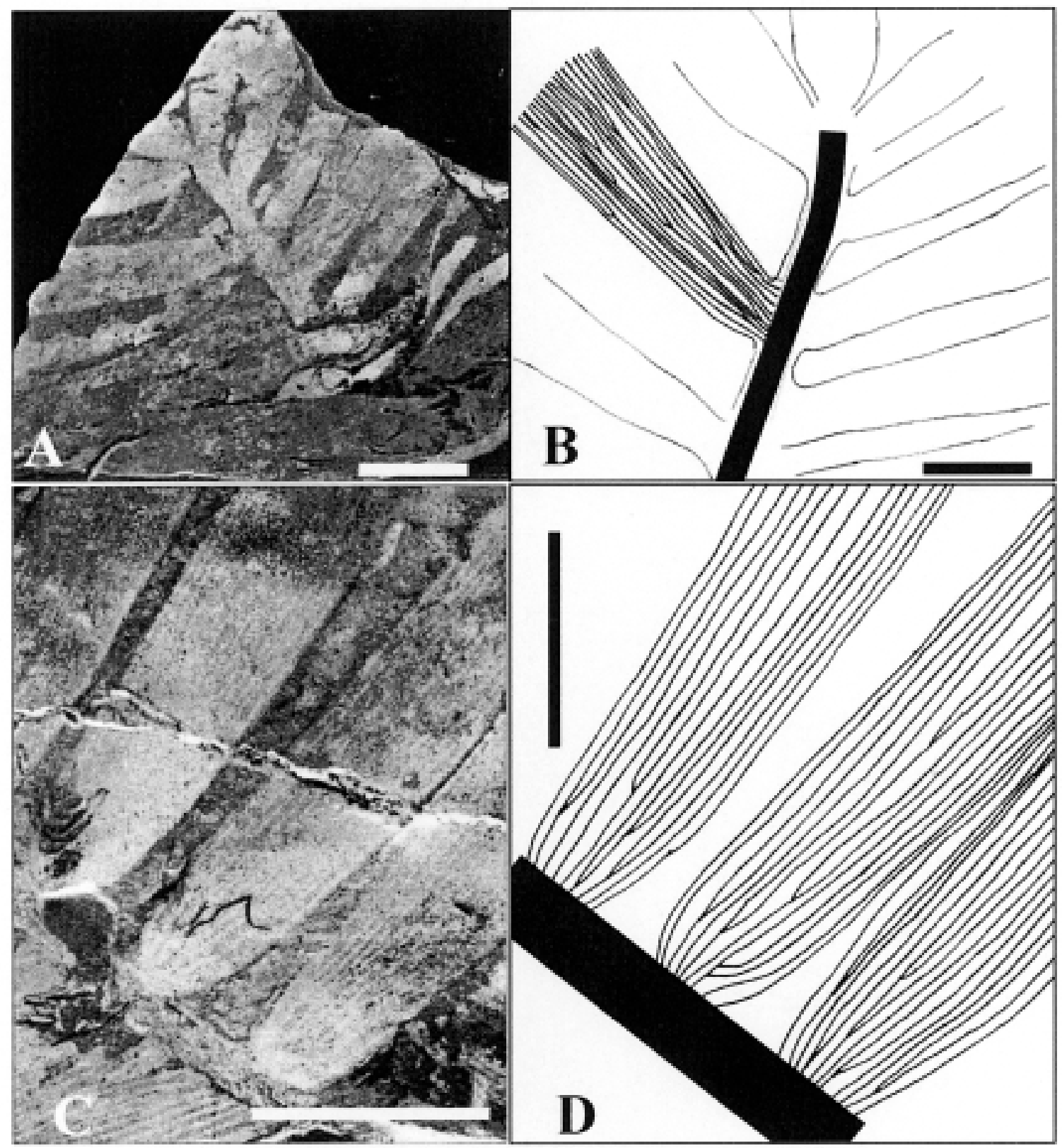

Fig. 4: (A, B) Pseudoctenis spatulata Du Toit (TriX 19), aspecto de la porción apical de la hoja (C, D), detalle de la venación con dicotomía. Barras de escala representan $1 \mathrm{~cm}$.

(A, B) Pseudoctenis spatulata Du Toit (TriX 19), aspect of the leaf apical portion; (C, D) detail of the venation with dichotomy. Scale bars represent $1 \mathrm{~cm}$ length.

basal de las frondas y de 4,5 a $5 \mathrm{~mm}$ en la porción distal. Del raquis emergen entre 8-11 venas oblicuas hasta aproximadamente la mitad inferior de la pinna, luego siguen paralelas a lo largo de la pinna. Se observa dicotomía de las venas predominantemente en la zona media de la pinna, terminando en alrededor de 19-21 venas en la parte distal. En el material estudiado no se observa ni longitud ni ápice de las pinnas.
Discusión: el material observado concuerda con los caracteres señalados por Artabe (1985) en Río Negro, Argentina, por Gnaedinger (1999) y por Anderson \& Anderson (1989), en lo que tiene relación con la forma de espátula de las pinnas, la inserción y disposición de estas en el raquis, la constricción basal y por último el número de venas en las pinnas que en su zona basal presenta de 8 a 11 venas, terminando en la porción distal entre 19-21 venas. 
Clase Cycadopsida Meyen, 1984.

Orden Cycadales Meyen, 1984.

Pseudoctenis truncata Leppe et P. Moisan, nov. sp. (Fig. 5A-D). Diagnosis: Fronda incompleta de $25 \mathrm{~mm}$ de largo y $65 \mathrm{~mm}$ de ancho, con ápice desconocido, las pinnas presentan ápice truncado. Raquis conservado de $2 \mathrm{~mm}$ de ancho. Pinnas de disposición opuesta y alternada, llegan a medir en el ejemplar hasta $50 \mathrm{~mm}$ de largo; en su zona basal presenta constricción de entre 15 a $20 \mathrm{~mm}$ de ancho, y en su zona distal mide entre 20 a $25 \mathrm{~mm}$ de ancho. Presenta un espaciamiento entre las pinnas en la zona basal de 25 a 30 $\mathrm{mm}$ de ancho, y en su zona distal llega a medir hasta $35 \mathrm{~mm}$ de ancho. Inserción de las pinnas entre 60 a $70^{\circ}$. Cada pinna presenta entre 7 a 8 venas, las que emergen oblicuas desde el raquis para terminar paralelas; no hay dicotomía.

Diagnosis: Incomplete frond of $25 \mathrm{~mm}$ long and $65 \mathrm{~mm}$ wide, unknown apex and pinnae with rounded truncate apex. Conspicuous rachis with $2 \mathrm{~mm}$ wide. The disposition of pinnae is alternate and opposite, with until $55 \mathrm{~mm}$ long, basal constriction between 15 and $20 \mathrm{~mm}$ wide and a distal wide between 20 to $25 \mathrm{~mm}$ Shows a distance between pinnae in the basal portion of about 25 to $30 \mathrm{~mm}$ wide, changing to $35 \mathrm{~mm}$ in the distal portion. Pinnae inserted laterally in angles between $60^{\circ}$ to $70^{\circ}$. Each pinnae exhibit 7 or 8 parallel veins, arising obliquous from the basis and without any dichotomy.

Holotipo: TriX 2. Localidad tipo: cota 100 de Quebrada La Mina, Cerro Calquinhue $(7 \mathrm{~km}$ al N de Quilacoya). Horizonte: Miembro Quilacoya de Formación Santa Juana.

Edad: Triásico Superior. Derivatio nominis: el epíteto específico truncata hace referencia a la peculiaridad del ápice de las pinnas, que a diferencia de casi la totalidad de las especies del género Pseudoctenis, presenta una forma truncada casi recta. Sin embargo, existe una especie con esta característica del ápice de las pinnas. Se trata de Pseudoctenis anomozamoides, descrita por Bonetti (1968) como hojas con pinnas de ápice truncado, algo redondeado. Las diferencias están dadas por la arquitectura foliar. Mientras Pseudoctenis anomozamoides Bonetti presen-

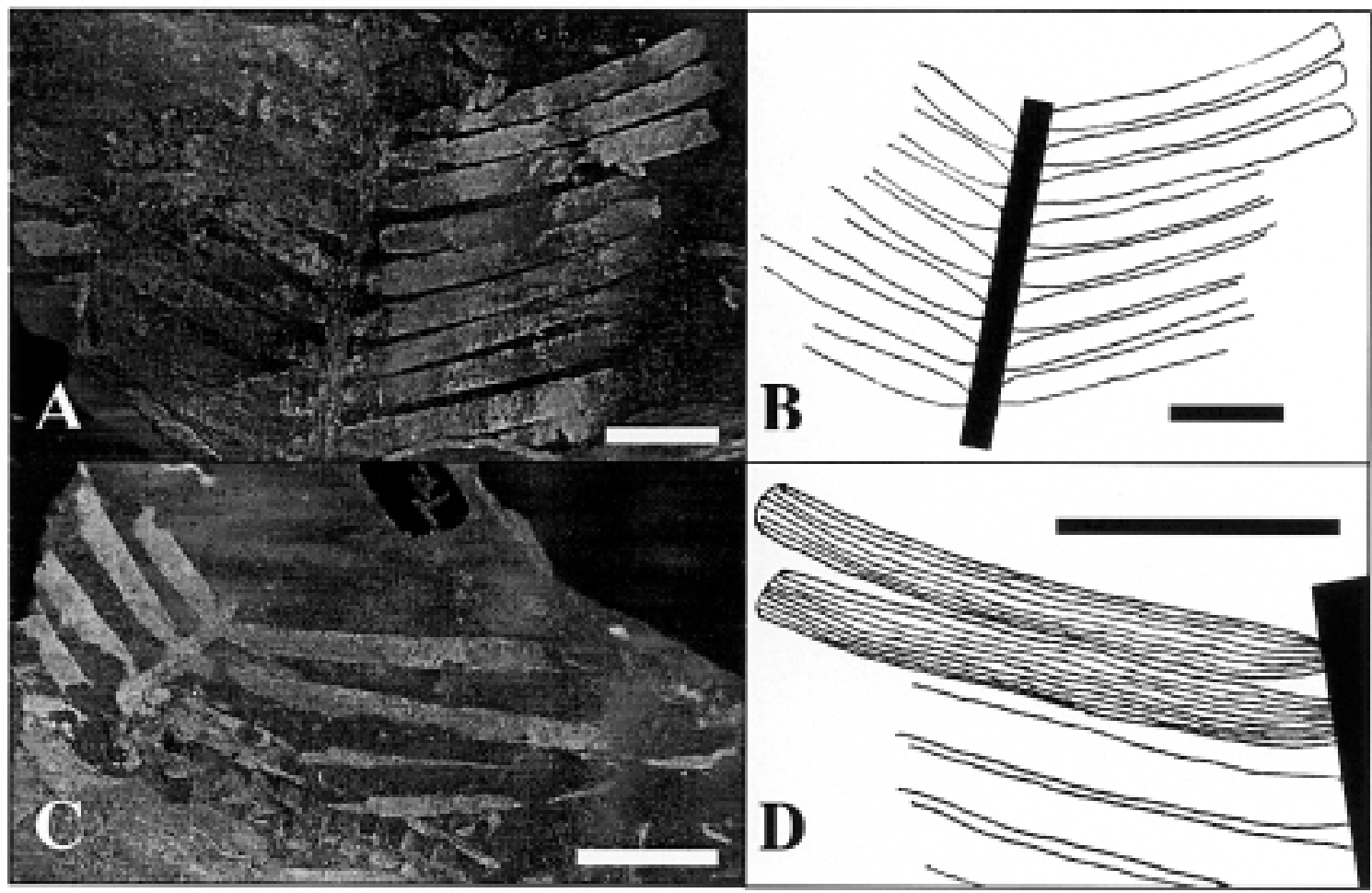

Fig. 5: (A, B) Pseudoctenis truncata nov. sp. holotipo (TriX 2); (C) probable fragmento de porción apical de hoja; (D) detalle de la venación del holotipo, mostrando la venación simple sin dicotomía y la inserción de las pinnas con una constricción en la base. Barras de escala representan $1 \mathrm{~cm}$.

(A, B) Pseudoctenis truncata nov. sp. holotype (TriX 2); (C) possible fragment of leaf apical portion; (D) detail of the holotype venation, showing simple venation without dichotomy and the insertion of the pinnae with basal constriction. Scale bars represent $1 \mathrm{~cm}$ length. 
ta venación con dicotomías de primer y segundo orden, $P$. truncata nov.sp. muestra venación simple, sin dicotomía alguna. La especie nueva Pseudoctenis truncata también presenta otros caracteres, como la ausencia de ala laminar, característica ampliamente presente entre las especies de Pseudoctenis. Pseudoctenis spatulata Du Toit y Pseudoctenis capensis $\mathrm{Du}$ Toit también comparten una morfología externa común con $P$. truncata, pero exhiben dicotomías en la venación de las pinnas y ala laminar en la inserción de las mismas. Diferencias importantes se pueden hallar a nivel del ápice de las pinnas, que para el caso de las especies de Pseudoctenis spatulata Du Toit y Pseudoctenis capensis es obtusamente redondeado (Gnaedinger 1999) ${ }^{1}$.

Discusión: Pseudoctenis truncata nov. sp. posee morfología similar a $P$. anomozamoides, $P$. capensis y $P$. spatulata. Sin embargo, posee una constricción basal y ausencia de ala laminar que no posee ninguna especie descrita del género Pseudoctenis. Además, se diferencia porque cada pinna posee de 7 a 8 venas en su porción basal, sin dicotomía, mientras que $P$. spatulata posee de 16 a 20 venas por pinna y $P$. capensis posee de 10 a 12 venas por pinna, ambas especies exhibiendo dicotomías en su venación. Las restantes especies de Pseudoctenis distan mucho más en cuanto a su morfología externa, señalada por Artabe (1985) en su clave para 28 especies del género.

Clase Cycadopsida Meyen, 1984.

Orden Cycadeoidales (Bennettitales) Meyen, 1984: Género Pterophyllum Brongniart, 1828: Especie tipo P. longifolium Brongniart, 1828. Pterophyllum azcaratei Herbst y Troncoso, 2000 (Fig. 6A-D). Material estudiado: TriX 1218 , recolectado en las localidades de Gomero y

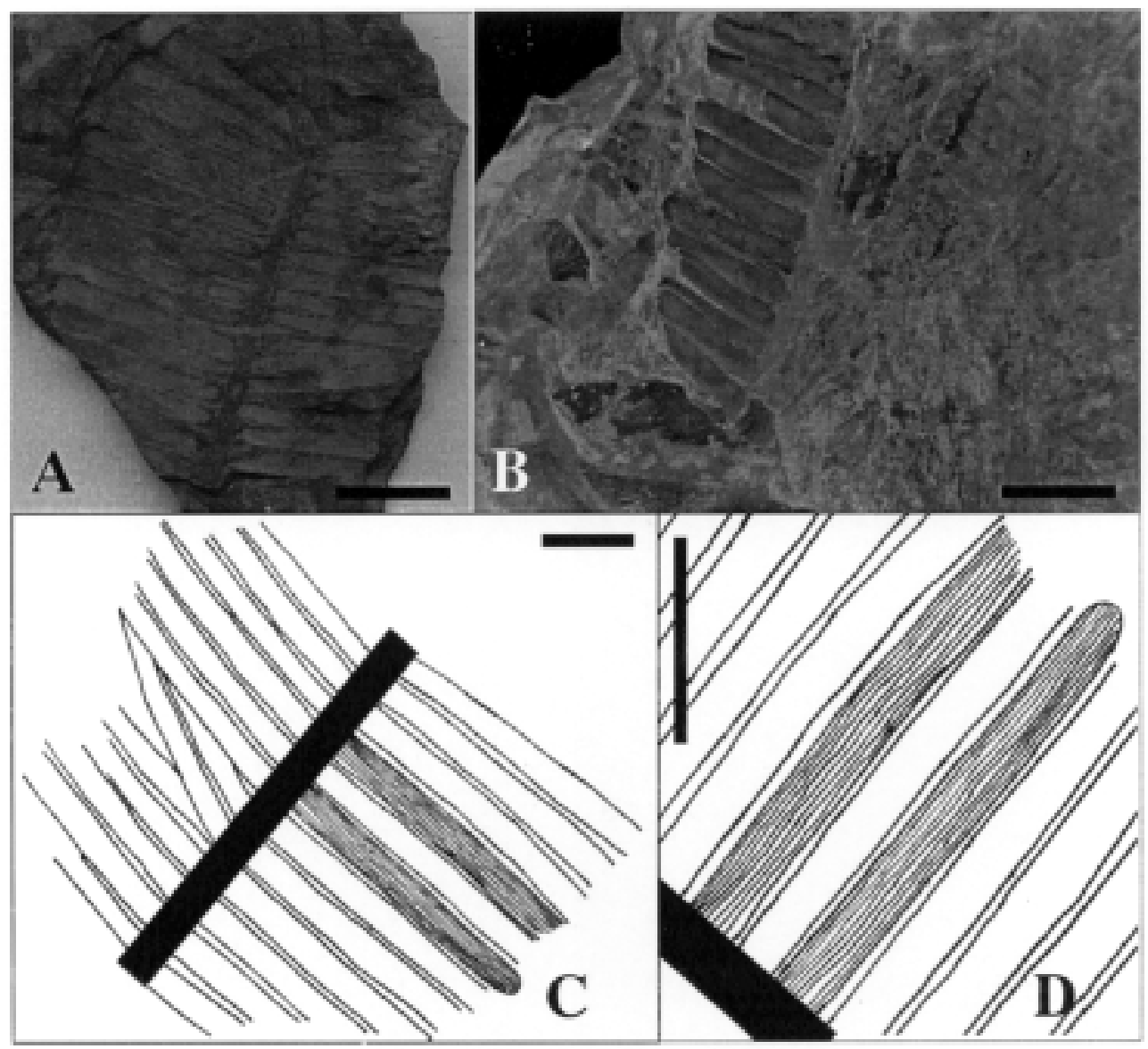

Fig. 6: (A, B) Pterophyllum azcaratei Herbst \& Troncoso (TriX 13-14), porción de hoja (C, D), aspecto general de la fronda con detalle de la venación sin dicotomía y las pinnas insertas lateralmente en el raquis por toda su base. Barras de escala representan $1 \mathrm{~cm}$.

(A, B) Pterophyllum azcaratei Herbst \& Troncoso (TriX 13-14), leaf portion; (C, D) general aspect of the leaf with detail of the venation without dichotomy and the pinnae inserted laterally on the rachis by the whole base. Scale bars represent $1 \mathrm{~cm}$ length. 
Cerro Calquinhue. Descripción: Frondas incompletas de hasta $170 \mathrm{~mm}$ de largo y de 60-80 $\mathrm{mm}$ de ancho. Raquis de 2 a $4 \mathrm{~mm}$ de ancho con una leve estriación. Las pinnas se insertan en el raquis en ángulos entre 80 y $90^{\circ}$, y a una distancia entre ellas de 1 a $1,5 \mathrm{~mm}$. No existe conexión entre las pinnas y estas llegan a medir $40 \mathrm{~mm}$ de largo y entre 3 a $5 \mathrm{~mm}$ de ancho. Las pinnas poseen bordes paralelos y rectos, sus bases son simétricas y sin arqueamiento basal ni ala laminar. El ápice de las pinnas es truncado, algo redondeado, y se insertan de forma opuesta, no observándose alternancia entre ellas. Presenta entre 11 y 13 venas en la parte basal, las cuales emergen rectas y paralelas hasta la parte distal; no se observa dicotomía.

Discusión: los ejemplares colectados se ajustan a las características enunciadas para la especie por Herbst \& Troncoso (2000). Se diferencia claramente del género Pseudoctenis por la ausencia de ala laminar y decurrencia en la porción basal de las pinnas. El género había sido reconocido para el sector de Buenuraqui-Gomero por Tavera (1960), sin que se llegara a nivel específico. Sin embargo, Herbst \& Troncoso (2000) señalan las similitudes del material estudiado por Tavera (1960) con la especie P. azcaratei. En la Región del Biobío la especie $P$. azcarate $i$ aparece asociada a rocas del Nórico-Rético.

\section{DISCUSIÓN}

La flora de la Formación Santa Juana se caracteriza por ser un afloramiento costero, con planicies estrechas cercanas a focos de actividad volcánica. Se diferencia de las floras Triásicas argentinas caracterizadas por ser montanas, con pequeñas cuencas lacustres (Leppe et al. 2000) $)^{2}$. Anderson \& Anderson (1983) sitúan climáticamente a Chile en el Cinturón Gondwánico Costero SW, definido por un invierno fuertemente templado lluvioso, un verano luminoso, cálido y húmedo, con vientos provenientes del Oeste conduciendo aire marino tierra adentro. La vegetación debía asemejarse a un bosque templado lluvioso. Anderson \& Anderson (1983) han caracterizado el Triásico de Concepción como compuesto por macrofloras desprovistas de cutícula.

La presencia de los géneros Pseudoctenis y Pterophyllum en distintos estratos de la Formación Santa Juana refuerza la idea de que existen distintas asociaciones paleoflorísticas dominando en diferentes momentos del amplio registro triásico del río Biobío. Las asociaciones se van sucediendo en forma abrupta y muchas veces no comparten elementos comunes entre unos y otros niveles. Lo anterior estaría estrechamente relacionado con el carácter costero o mediterráneo de las floras en sentido de Retallack (1987), manifestado fitosociológicamente como la dominancia de la asociación Linguifolietum en ambientes anegados y de fuerte influencia costera, y la asociación Dicroidietum odontopteroidium en ambientes más continentales, con sedimentación lacustre o de otros cuerpos de agua dulce. También se ve ratificado por el tipo de sedimentación evidenciado por las rocas, que en el caso de las llanuras costeras se ven constantemente afectadas por transgresiones marinas que dejan restos de invertebrados marinos como Halobia en estratos alternados con restos vegetales de la asociación dominada por Linguifolium, Heidiphyllum y Pseudoctenis. Dicroidium (Anexo 1), por otro lado, se hace mucho más frecuente en las asociaciones del interior continental. Finalmente, la existencia de estas discontinuidades paleoflorísticas no hace más que reforzar la idea de que la Formación Santa Juana no es una unidad litológica, estratigráfica y menos paleontológica, aunque estudios anteriores de los autores parecen indicar que dichas secuencias corresponderían a diferentes pisos del Triásico Superior, entre el Cárnico y el Nórico con certeza, pues aún se discute la presencia del Rético. Según diversos autores, el término Rético solo puede ser usado correctamente cuando se encuentran capas transicionales entre el Triásico más superior y el Liásico más inferior, en un continuo sin hiatos paleontológicos ni litológicos (Herbst 1965).

La especie nueva Pseudoctenis truncata viene a complementar el conocimiento de las $\mathrm{Cy}$ cadales y Cycadeoidales representada por las especies Pseudoctenis longipinnata Anderson \& Anderson, Pseudoctenis spatulata Du Toit y Pterophyllum azcaratei Herbst \& Troncoso. Esta nueva especie se diferencia claramente de las especies del género Pseudoctenis señaladas para el Triásico de Argentina y Chile, principalmente en base a su filotaxia. La correcta determinación de los taxa presentes en el Triásico de la Región del Biobío resulta fundamental para establecer cuánto se ajustan la realidad a los modelos paleofitosociológicos formulados para el Gondwana, y como un aporte al conocimiento de la evolución de grupos que teniendo un conjunto de caracteres convergentes, enfrentaron los periodos de extinción masiva mesozoica de distinta forma, significando en el caso de las Cycadales una selección que dejó como remanentes actuales a las Cycadaceas con hoja pinnatifoliada, y para el caso de las Cycadeoidales (Bennettitales) significó la extinción total 
durante el Cretácico. La condición de borde continental en que se situaba la Región del Biobío durante el Gondwana triásico, convierte a los afloramientos estudiados en una de las pocas evidencias importantes de estos taxa en el occidente del supercontinente.

\section{AGRADECIMIENTOS}

Los autores agradecen a la Dra. Analía Artabe (Museo de La Plata, Argentina) por su valiosa colaboración, así como a la Profesora Sylvia Palma-Heldt, por su apoyo desinteresado y al Proyecto FONDECYT 2010105 por financiar el presente trabajo.

\section{LITERATURA CITADA}

ANDERSON JM \& HM ANDERSON (1983) Paleoflora of Southern Africa. Molteno Formation (Triassic), Volume 1, Part 1. Introduction, Part 2. Dicroidium. A.A. Balkema, Rotterdam, The Netherlands. 227 pp.

ANDERSON JM \& HM ANDERSON (1989) Paleoflora of Southern Africa. Molteno Formation (Triassic), Volume 2: Gymnosperms (excluding Dicroidum). A.A. Balkema, Rotterdam, The Netherlands. 567 pp.

ARTABE AE (1985) Estudio sistemático de tafoflora triásica de Los Menucos, Provincia de Río Negro, Argentina. Parte II. Cycadophyta, Ginkgophyta, Coniferophyta. Ameghiniana 22: 159-180.

ARTABE AE \& DW STEVENSON (1999) Fossil Cycadales of Argentina. Botanical Review 65: 219-238.

BONETTI MIR (1968) Las especies del género Pseudoctenis en la flora triásica de Barreal (San Juan). Ameghiniana 5: 433-446.

BRONGNIART A (1828) Historie des vegetaux fossils, ou recherches botaniques et geologiques sur les vegetauxrenfermès dans les diverses couches du globe, I (1828-1837), XII. Paris, France. 488p.

BRÜGGEN J (1934) Gründzuge der Geologie und Lagerstättenkunde Chiles. MathematischNaturwissenschaflitche Klasse der Heidelberger Akademie der Wissenschaften, Germany. $362 \mathrm{pp}$.

CRANE P (1985) Phylogenetic analysis of seed plants and the origin of angiosperms. Annals of the Missouri Botanical Garden 72: 716-793.

CUCURELLA J (1978) Estudio geológico y radiométrico del valle inferior del río Biobío, Provincia de Concepción, VIII Región. Memoria para optar al título de Geólogo, Departamento. de Geología, Facultad de Ciencias Físicas y Matemáticas, Universidad de Chile, Santiago, Chile. 114 pp.

DU TOIT AL (1927) The fossil flora of the Upper Karroo beds. Part II. Annals of South African Museum 22 289-420.
FOSTER AS \& EM GIFFORD (1989) Comparative morphology of vascular plants. W.H. Freeman and Company, San Francisco, California. 524 pp.

FUENZALIDA H (1937) El Rético en la costa de Chile central. Departamento de Minas y Petróleo (Chile) 6: 739-747.

GERTH H (1935) Geologie Südamerikas. Zweiter Teil Gebrüder Borntraeger, Berlin, Germany. 389 pp.

GIFFORD E \& A FOSTER (1987) Morphology and evolution of vascular plants. Third Edition. W. H. Freeman and Company, New York, New York, USA. $626 \mathrm{pp}$.

GROEBER P (1934) Pérmico y Triásico en la costa de Chile. Boletín de Mineralogía y Petrología (Chile) 5: $510-513$.

HERBST R (1965) La flora fósil de la formación Roca Blanca, Provincia de Santa Cruz, Patagonia. Opera Lilloana (Argentina) 12: 7-101.

HERBST R \& A TRONCOSO (2000) Las Cycadophyta del Triásico de las formaciones La Ternera y El Puquén (Chile). Ameghiniana 37: 283-292.

JAWORSKI E (1922) Die Marine Trias in Südamerika. Neiues Jahrbuch für Mineralogie. Geologie und Paläontologie (Beilage Bundel) 47: 93-200.

KUMMEL B \& RL FUCHS (1953) The Triassic of South America. Boletín de la Sociedad de Geología del Perú 26: 95-120.

MEYEN B (1984) Basic features of Gymnosperms: systematics and phylogeny as evidenced by the fossil record. Botanical Review 50: 1-111.

NIELSEN S (1999) Die triassche Santa Juana Formation am Unterlauf des Río Bíobío, Chile. Diplomarbeit und Diplomkartierung Im Fach GeologiePaläontologie an der Universität Hamburg, Hamburg, Germany. 84 pp.

NISHIDA $\mathrm{H}$ (1994) Morphology and evolution of Cycadeoidales. Journal of Plant Research 107: 479-492.

RETALLACK GJ (1987) Triassic vegetation and geography of the New Zealand portion of the Gondwana supercontinent. Monograph by the American Geophysical Union, Washington, District of Columbia, USA. 252 pp.

SEWARD AC (1911) the Jurassic flora of Sutherland. Transactions of the Royal Society of Edinburgh. 47: 663-709.

STEINMANN G (1921) Rhätischen Floren und Landverbindungen auf der Südhalbkugel. Geologische Rundschau. Band XI. Leipzig, Germany. $56 \mathrm{pp}$

STEWART WN \& GW ROTHWELL (1993) Paleobotany and the evolution of plants. Second edition. Cambridge University Press, Cambridge, United Kingdom. 521 pp.

TAVERA J (1960) El Triásico del valle inferior del río Biobío. Universidad de Chile, Instituto de Geología (Chile) 18: 321-345.

TAYLOR TN \& EL TAYLOR (1993) The biology and evolution of fossil plants. Prentice-Hall, Englewood Cliffs, New Jersey. 324 pp.

VEYL C (1961) Contribución al conocimiento de la geología regional de la Provincia de Concepción. Revista del Instituto de Ingeniería en Minas de Chile 72: 1-51. 


\section{ANEXO 1}

Listado de géneros de plantas fósiles frecuentemente hallados junto a Cycadales y Cycadeoidales en el Triásico superior del Biobío

List of fossil plant genera frequently found close to Cycadales and Cycadeoidales in the Upper Triassic of Biobío

Asterotheca

Taeniopteris

Saportaea

Yabeiella

Linguifolium

Jungites

Baiera

Sphenobaiera

Heidiphyllum

Dejerseya

Clathropteris
Gleichenites

Gontriglossa

Cladophlebis

Neocalamites

Dictyophyllum

Dicroidium

Pachydermophyllum

Pteruchus

Umkomasia

Kurtziana

Thaumatopteris 